\title{
Investigation of hydrogenous materials using neutrons
}

\author{
B A DASANNACHARYA and P S GOYAL \\ Nuclear Physics Division, Bhabha Atomic Research Centre, Bombay 400 085, India
}

\begin{abstract}
Slow neutrons have proved to be a very powerful probe for examining materials in general and hydrogenous materials in particular. In this article we review various neutron scattering techniques which have been utilised to investigate different aspects of a variety of hydrogenous materials. Translationally as well as orientationally disordered materials, ferroelectrics, superconductors, metal-hydrogen systems, polymers and biological molecules have been chosen as illustrative examples.
\end{abstract}

Keywords. Neutrons; hydrogenous materials; ferroelectrics; disordered materials; superconductors; metal-hydrogen systems; polymers; biological molecules.

\section{Introduction}

Hydrogen is the most predominant element in nature. It behaves in very different ways in different environments thus giving rise to types of materials which show an extremely wide variety of properties. Not surprisingly, every possible method has been used to examine them. During the last quarter of a century neutron scattering techniques have made a very important contribution in the investigation of these materials. This is because of certain unique properties of slow neutrons which make them particularly suitable for such studies.

Neutron scattering consists of a family of techniques which give a host of complementary information about any substance. Coherent scattering (diffraction) of neutrons is well known for the information it gives on the crystallographic structures of hydrogenous materials; incoherent elastic scattering which is less commonly used can be particularly useful in studying Debye-Waller factor of hydrogen and the 'elastic' form factor which is connected with hydrogen atom distribution over a very large time interval; coherent inelastic scattering experiments determine phonon dispersion curves, whereas incoherent inelastic and quasi-elastic scattering measurements lead to phonon frequency distribution functions and give the nature of random atomic motions (translational and/or rotational) in solids and liquids; small angle scattering of long wave-length neutrons leads to information on large molecules (polymers or biomolecules) or congregation of molecules (precipitates etc). Most of the above mentioned techniques have been used extensively at Trombay during the last two decades. This overview will, with some representative examples, endeavour to bring out the scope of these neutron techniques, particularly as applied in connection with hydrogenous materials.

\section{Principles}

\subsection{Basic interaction}

Slow neutrons which typically have wavelength in the range of 1 to $10 \AA$ are mainly 
scattered from the nucleus of atoms*. The scattering, being essentially from a point scatterer $\left(\sim 10^{-13} \mathrm{~cm}\right)$, is isotropic. The extent of scattering, which is proportional to the square of the bound nuclear scattering amplitude, $b$, varies for any element from one isotope to another and also depends on the spin of the scattering nucleus. Hence in a random assembly of atoms a configurational average, $\langle\cdots\rangle$, has to be taken. This gives rise to a 'coherent' scattering cross-section, $\sigma_{\text {coh }}=4 \pi\langle b\rangle^{2}$ where the intensity is proportional to the square of the average scattering amplitude and an incoherent scattering which is the difference between the total, $4 \pi\left\langle b^{2}\right\rangle$, and the coherent scattering; $\sigma_{\text {inc }}=4 \pi\left[\left\langle b^{2}\right\rangle-\langle b\rangle^{2}\right]$. Table 1 gives the coherent and incoherent scattering cross-sections and scattering amplitudes for some elements.

Several features of this table are worth noting: $\sigma_{\text {inc }}$ for $\mathrm{H}$ practically dominates all scattering, $\sigma_{\text {coh }}$ for $\mathrm{H}$ is comparable to that of other elements and the scattering amplitudes of $\mathrm{H}$ is negative whereas most other elements have a positive value of $b$. These along with the other properties of neutrons, that is, their high penetrability in materials and wavelength-energy $(\lambda-\omega)$ relationship similar in order-of-magnitude to that of material lattices are utilised in most neutron scattering experiments.

A typical block diagram for a neutron scattering experiment is shown in figure 1. A neutron source, usually a nuclear reactor provides a continuous source of polychromatic neutrons. A monochromator which may be just a large single crystal or a more complicated mechanical device, is used to get a mono-energetic beam of neutrons of energy $E_{0}$ having a wavelength $\lambda_{0}$ and wave-vector $\mathbf{k}_{0} ;\left|k_{0}\right|=2 \pi / \lambda_{0}, E_{0}=\hbar^{2} k_{0}^{2} / 2 m$. These neutrons are scattered from the material under study. In a diffraction experiment

Table 1.

\begin{tabular}{cccc}
\hline & $\sigma_{\mathrm{coh}}\left(10^{-24} \mathrm{~cm}^{2}\right)$ & $\sigma_{\mathrm{Inc}}\left(10^{-24} \mathrm{~cm}^{2}\right)$ & $b\left(10^{-12} \mathrm{~cm}\right)$ \\
\hline $\mathrm{H}$ & 1.759 & 79.7 & -0.3741 \\
$\mathrm{D}$ & 5.597 & 2.0 & 0.6674 \\
$\mathrm{C}$ & 5.554 & 0.0 & 0.6648 \\
$\mathrm{O}$ & 4.235 & 0.0 & 0.5805 \\
$\mathrm{Fe}$ & 11.437 & 0.21 & 0.954 \\
$\mathrm{~Pb}$ & 11.106 & 0.0 & 0.9401 \\
\hline
\end{tabular}

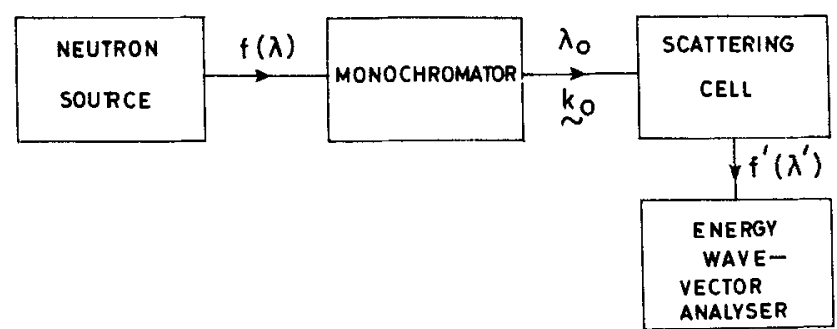

Figure 1. Schematic drawing of a neutron scattering experimental set-up.

\footnotetext{
* Materials having a magnetisation distribution also show a magnetic scattering which may be comparable to nuclear scatterings; we will not consider such substances here.
} 
one measures the angular distribution of the scattered neutrons. In a more general inelastic or quasi-elastic scattering experiment both the angular (giving wavevector transfer, $\mathbf{Q}$ ) and energy distributions are measured. Thus, in the latter the scattering cross-section for wavevector transfer $\mathbf{Q}=\mathbf{k}_{0}-\mathbf{k}^{\prime}$ and energy transfer, $\varepsilon=\hbar \omega=E_{0}$ $-E^{\prime}$, are determined.

For convenience, we collect the relevant expressions for cross-sections here (Kostorz and Lovesey 1979). The examples of their use will form the subject matter of the rest of this article.

\subsection{Diffraction: Coherent scattering}

The angular distribution of coherently scattered neutrons per unit volume of the sample is given by:

where

$$
(\mathrm{d} \sigma / \mathrm{d} \Omega)_{\operatorname{coh}}=N_{0} \frac{(2 \pi)^{3}}{V_{0}} \sum_{\tau} \delta(\mathbf{Q}-\tau)|F(\tau)|^{2},
$$

$$
F(\tau)=\sum_{i}\left\langle b_{l}\right\rangle \exp \left(i \tau \cdot \mathbf{r}_{l}\right) \cdot \exp \left\{-W_{l}(\tau)\right\}
$$

Here $\mathbf{r}_{l}$ denotes the co-ordinates of $l$ th nucleus in the unit cell, $\tau$ the reciprocal lattice vector and $\exp \{-W(\tau)\}$ the Debye-Waller factor for the $I$ th nucleus. $V_{0}$ is the volume of the unit cell and $N_{0}$ is the number of unit cells per unit volume of the sample. The $\delta$-function in (1) signifies that intensity appears only at angles satisfying the Bragg reflection condition $\mathbf{Q}=\tau$. In a crystallographic experiment, the quantity of interest is the structure factor $|F(\tau)|^{2}$.

\subsection{Diffraction: Incoherent scattering}

The angular distribution of intensity of incoherently scattered neutrons is given by:

$$
(\mathrm{d} \sigma / \mathrm{d} \Omega)_{\mathrm{inc}}=N_{0} \sum_{l} \frac{\sigma_{\mathrm{inc}}^{l}}{4 \pi} \exp \left\{-2 W_{l}(Q)\right\} .
$$

This intensity, in hydrogenous materials, for all practical purposes is due to hydrogen alone (due to its large $\sigma_{\text {inc }}$ ) and one measures essentially the Debye-Waller factor of the hydrogen atoms.

\subsection{Inelastic coherent scattering: One phonon process}

The coherent one-phonon scattering cross-section per unit volume for neutron energy loss is given by:

$$
\begin{aligned}
\left(\mathrm{d}^{2} \sigma / \mathrm{d} \Omega \mathrm{d} E^{\prime}\right)_{\substack{\text { Coh. } \\
\text { inel }}=} & \frac{k^{\prime}}{k_{0}} \frac{(2 \pi)^{3}}{V_{0}} \sum_{\tau, j, q}\left|G_{j}(\mathbf{q}, \mathbf{Q})\right|^{2} \frac{\left[n_{j}(\mathbf{q})+1\right]}{\omega_{j}(\mathbf{q})} \\
& \times \delta\left[\omega-\omega_{j}(\mathbf{q})\right] \delta(\mathbf{Q}-\mathbf{q}-\tau),
\end{aligned}
$$

where the structure factor is

$$
G_{j}(\mathbf{q}, \mathbf{Q})=\frac{1}{\left(2 M_{l}\right)^{1 / 2}} \sum_{l}\left\langle b_{l}\right\rangle\left\{\mathbf{Q} \cdot \mathbf{e}_{j}^{l}(\mathbf{q})\right\} \exp \left(i \mathbf{Q} \cdot \mathbf{r}_{l}\right) \exp \left\{-W_{l}(\mathbf{Q})\right\}
$$

Here $q$ is the wavevector of the phonon being excited and $j$ its polarization index. $\omega_{j}(\mathbf{q})$ 
is the angular frequency of the mode and $\mathbf{e}_{j}^{l}(\mathbf{q})$ its polarisation vector for $l$ th atom whose mass is $M_{l}$. $\left(n_{j}(q)+1\right)$ is the polulation factor. Here both energy and wavevector conservation conditions have to be satisfied through the two- $\delta$-functions. This relation is utilized for determining phonon dispersion curves.

\subsection{Inelastic incoherent scattering: One phonon process}

For incoherent scattering, scattering cross-section for unit volume of the sample is given by:

$$
\begin{aligned}
\left(\frac{\mathrm{d}^{2} \sigma}{\mathrm{d} \Omega \mathrm{d} E^{\prime}}\right)_{\mathrm{incl}}= & \frac{k^{\prime}}{k_{0}} \sum_{l j q}\left(\frac{\sigma_{\text {inc }}^{l}}{4 \pi}\right) \frac{\left[n_{j}(\mathbf{q})+1\right]}{2 M_{l} \omega_{j}(\mathbf{q})}\left|\mathbf{Q} \cdot \mathbf{e}_{j}^{l}(\mathbf{q})\right|^{2} \\
& \times \exp \left\{-2 W_{l}(\mathbf{Q})\right\} \delta\left(\omega-\omega_{j}(\mathbf{q})\right) .
\end{aligned}
$$

The cross-section now depends on the number of modes satisfying the energy conservation condition. This gives a polarisation weighted phonon frequency distribution function. Incoherent inelastic scattering is particularly useful in examining phonon spectra of hydrogenous materials.

\subsection{Quasi-elastic incoherent scattering: Diffusive motion}

Random dynamical motions such as molecular reorientations and diffusion give rise to a line broadening of the incident monoenergetic beam. This is referred to as quasielastic scattering. In the case of a hydrogen atom translationally diffusing in a solid one has (Springer 1972)

$$
\left(\frac{\mathrm{d}^{2} \sigma}{\mathrm{d} \Omega \mathrm{d} E^{\prime}}\right)_{\substack{\text { inc } \\ \mathbf{q} \cdot \mathrm{el}}}=\frac{N \sigma_{\text {inc }}}{4 \pi^{2} \hbar} \frac{k^{\prime}}{k_{0}} \exp \{-2 W(Q)\} \sum_{p} g_{p}(Q) \frac{f_{p}(Q)}{\omega^{2}+f_{p}^{2}(Q)},
$$

with the normalising condition $\sum_{p} g_{p}(Q)=1$. Here $N$ is the number of hydrogen atoms per unit volume. The widths, $f_{p}(Q)$ of the Lorentzians are decided by the diffusion coefficient, and $g_{p}(Q)$ is related to the actual geometry of jump diffusion.

The incoherent scattering from reorienting molecular groups, like $\mathrm{NH}_{4}, \mathrm{CH}_{3}$ etc, is given by

$$
\begin{aligned}
\left(\frac{\mathrm{d}^{2} \sigma}{\mathrm{d} \Omega \mathrm{d} E^{\prime}}\right)_{\mathrm{q} \cdot \mathrm{el} .}= & \frac{N \sigma_{\text {inc }}}{4 \pi^{2} \hbar} \frac{k^{\prime}}{k_{0}} \exp \{-2 W(Q)\} \\
& \times\left[\pi A(Q) \delta(\omega)+\sum_{p} g_{p}^{\prime}(Q) \frac{f_{p}(Q)}{\omega^{2}+f_{p}^{2}(Q)}\right]
\end{aligned}
$$

with the normalising condition $\sum_{p} g_{p}^{\prime}(Q)+A(Q)=1$. The relative intensities of the elastic and quasielastic parts (form factor) are dependent on the geometry of molecular reorientation. The width of the Lorentzian is related to the reorientation rate. Incoherent elastic scattering experiment $(\$ 3.2)$ will usually examine the first term in (6). However, the measured intensities can have a small contribution from the second term also depending on the relative values of $f_{p}(Q)$ and the instrumental resolution used in such an elastic scattering experiment.

\subsection{Small angle neutron scattering: Molecular form factor}

Whenever the object of scattering, say, a polymer or a precipitate, is much larger than the wavelength of the radiation scattered, the scattering is confined to small angles. 
Hence special techniques have been developed for studying large scatterers (size typically $>50 \mathrm{~A}$ ). The technique basically depends on the contrasts in scattering between the object under study and the matrix holding this object. Fortunately for neutrons, since the scattering amplitude for hydrogen and deuterium is very different, it is possible to produce good contrast in scattering. The angular distribution is given by (King 1980).

$$
(\mathrm{d} \sigma / \mathrm{d} \Omega)_{\mathrm{SAS}}=N_{c} n^{2}\left(b_{s}-b_{h}\right)^{2} f(Q)
$$

Here $N_{c}$ is the number density of large molecules and $n$ is the number of atoms in the molecule, $b_{s}$ and $b_{h}$ are the average scattering amplitudes of the scatterer and the host and $f(Q)$ is a form factor associated with the molecule.

\section{Experimental results}

\subsection{Structures by coherent scattering}

Neutron diffraction as a crystallographic tool is particularly suitable for the accurate determination of the positions of hydrogen atoms, in contrast to the more commonly used $\mathrm{x}$-ray diffraction, since the scattering amplitude for hydrogen is comparable to that of most other elements. Literature is full of examples of inorganic, organic and biologically interesting structures determined using neutron diffraction (Dachs 1978) and it would be impossible even to give a flavour of the variety of such studies. We content ourselves by giving an example of a simple compound, $\left(\mathrm{NH}_{4}\right)_{2} \mathrm{CuCl}_{4} \cdot 2 \mathrm{H}_{2} \mathrm{O}$, which shows some subtle structural features in relation to the two ammonium ions in the molecule.

The framework of $\left(\mathrm{NH}_{4}\right)_{2} \mathrm{CuCl}_{4} \cdot 2 \mathrm{H}_{2} \mathrm{O}$ is made up of columns of $\mathrm{NH}_{4} \mathrm{Cl}$ and $\mathrm{CuCl}_{2} \cdot 2 \mathrm{H}_{2} \mathrm{O}$ along $c$ direction; the columns alternate along $a$ and $b$ directions. The cube of $\mathrm{NH}_{4} \mathrm{Cl}$ is rather similar to that of pure $\mathrm{NH}_{4} \mathrm{Cl}$, with $\mathrm{NH}_{4}^{+}$residing on a tetrahedral site surrounded by eight $\mathrm{Cl}$ ions, four of type-I and four of type-II (figure 2). Infra-red absorption experiments (Oxton and Knopp 1977) and Raman scattering experiments (Bansal et al 1979) alternatively suggested that $\mathrm{NH}_{4}^{+}$ions are ordered with $\mathrm{N}-\mathrm{H}$ bond pointing towards $\mathrm{Cl}(\mathrm{I})$ and that they are disordered. Neutron diffraction experiments (Bhakay-Tamhane et al 1980) not only unequivocally decided that they were disordered but could quantitatively assign the site occupancy ratio as $0.64: 0.36$ in favour of $\mathrm{Cl}(\mathrm{I})$ as shown in figure 2, a task not easily achieved by any other method. However, one notes that diffraction experiment itself is not able to answer whether this disorder is static or dynamic. We will address ourselves to this question presently.

\subsection{Hydrogen diffusion, reorientations through incoherent diffraction and incoherent 'elastic' scattering}

In a diffraction experiment the coherent Bragg peaks which are measured to determine the structure, ride over an incoherent uniformly varying intensity which is very large for hydrogenous substances and is almost entirely due to incoherent scattering from hydrogens. The angular variation of this intensity is given by exp $-Q^{2}\left\langle u^{2}\right\rangle$ where $\left\langle u^{2}\right\rangle$ is the mean squared amplitude of the hydrogen atoms, assuming harmonic vibrations. When only vibrations are present this gives an accurate measure of $\left\langle u^{2}\right\rangle$. 

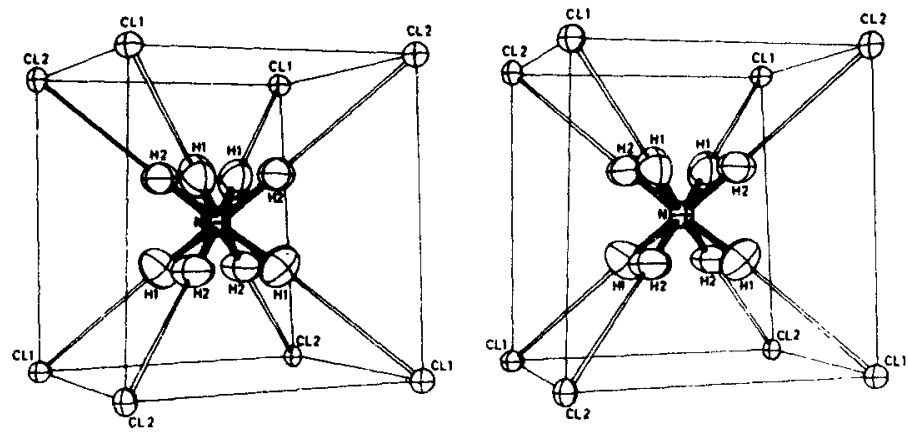

Figure 2. An illustration of surroundings of ammonium ions in $\left(\mathrm{NH}_{4}\right)_{2} \mathrm{CuCl}_{4} \cdot 2 \mathrm{H}_{2} \mathrm{O}$ at room temperature. $64 \% \mathrm{NH}_{4}^{+}$ions have orientation corresponding to $\mathrm{H}(1)$ and $36 \%$ corresponding to $\mathbf{H}(2)$. The thermal ellipsoids shown in this figure have been scaled to include $30 \%$ probability (Bhakay-Tamhane et al 1980).

As described earlier (\$2.6), it is also possible to do incoherent 'elastic' scattering experiment instead of incoherent diffraction. In such an experiment only the 'elastic' component of the scattered neutrons is measured. In an ideal 'elastic' scattering experiment one observes the time average, over infinite time, of the motion of the hydrogen atom. In real life experiment this averaging is qualitatively over a time $\Delta t=h / \Delta E$ where $\Delta E$ is the energy resolution of the 'elastic' scattering experiment. Thus, whenever in addition to vibrational motion the hydrogen atom also performs diffusive translational movements (as in metal-hydrogen systems) or large reorientational jumps (as in plastic solids, liquids or molecular crystals) the 'elastic' incoherent intensity depends on the characteristics of the diffusion times and distances.

Results of such an experiment on $\mathrm{NH}_{4} \mathrm{Br}, \mathrm{NH}_{4} \mathrm{I},\left(\mathrm{NH}_{4}\right)_{0.16} \mathrm{~K}_{0.84} \mathrm{I}$ and $\left(\mathrm{NH}_{4}\right)_{0.16} \mathrm{~K}_{0.84} \mathrm{Br}$ are shown in figure 3 (Bragg peaks are not shown for the sake of clarity) (Goyal and Dasannacharya 1979). In the case of $\mathrm{NH}_{4} \mathrm{Br}$ one finds that the distribution (figure 3a) with an elastic resolution of $12 \mathrm{meV}(x)$ is the same as that with a resolution of $1.3 \mathrm{meV}(\mathrm{o})$, showing that the reorientation times of $\mathrm{NH}_{4}^{+}$ions in the room temperature phase $\left(\mathrm{CsCl}\right.$ phase) of $\mathrm{NH}_{4} \mathrm{Br}$ are much larger than about $h / \Delta E=h / 1.3 \mathrm{meV} \sim 3 \times 10^{-12} \mathrm{sec}$. Consequently the angular distribution gives $\left\langle u^{2}\right\rangle$ which is $0.04 \AA^{2}$. The angular distribution in the other three examples, however, shows a much steeper non-gaussian fall, clearly indicating a rapid reorientation of tetrahedral $\mathrm{NH}_{4}^{+}$ions in their first neighbour octahedral environment. Assuming a model for the geometry of reorientation it was found that, $\tau$, the reorientation time (i.e. the time between two reorientational jumps) is $\sim 5 \times 10^{-12} \mathrm{sec}$ and depends only very weakly on temperature between 300 and $140^{\circ} \mathrm{K}$.

Similar measurements at a fixed angle but as a function of temperature can reveal the existence of phase transitions (de Graff 1980) and can also throw light on temperature dependence of diffusion coefficient (Leadbetter et al 1976). They are rather similar in nature to the line width measurements in NMR. While these measurements can be used to make a quick tour through the momentum-transfer to temperature space, they are not capable of giving detailed spatial information regarding the nature of the motion of hydrogen. For this one resorts to quasi-elastic scattering which is unique in this respect. 


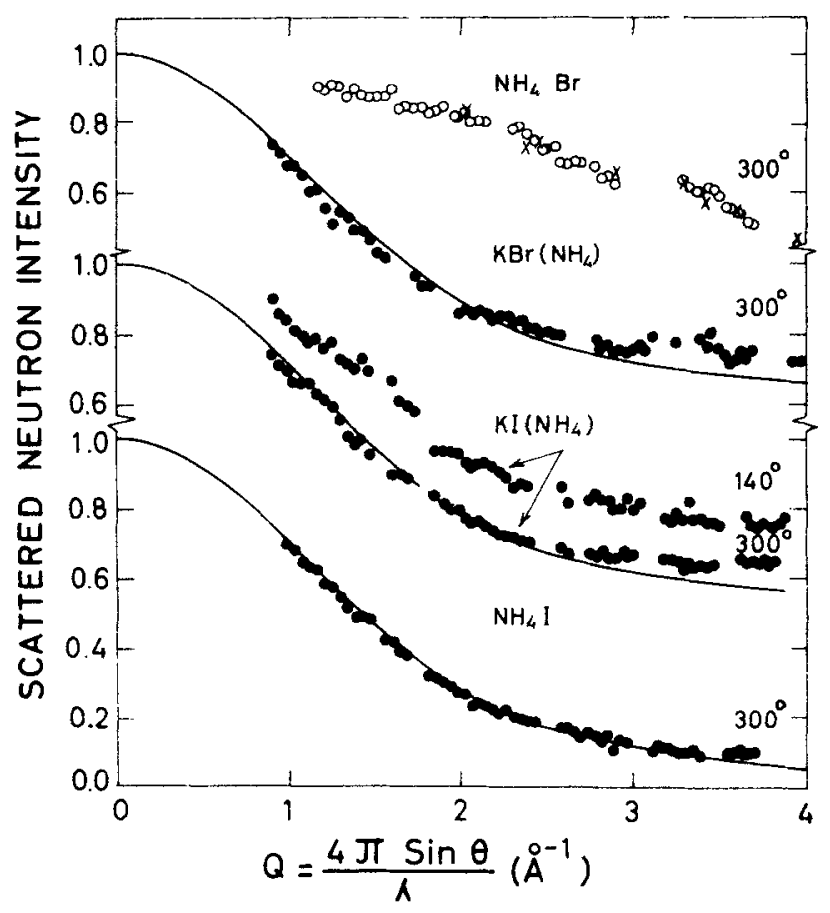

Figure 3. Scattered neutron intensity versus wavevector transfer for various salts as obtained from neutron incoherent "elastic" scattering experiment. (a) $\mathrm{NH}_{4} \mathrm{Br}$ at $300 \mathrm{~K}$ (b) $\left(\mathrm{NH}_{4}\right)_{0.16} \mathrm{~K}_{0.84} \mathrm{Br}$ at $300 \mathrm{~K}$ (c) $\left(\mathrm{NH}_{4}\right)_{0.16} \mathrm{~K}_{0.84} \mathrm{I}$ at $140 \mathrm{~K}$ (upper curves) and $300 \mathrm{~K}$ (lower curves) and (d) $\mathrm{NH}_{4} \mathrm{I}$ at $300 \mathrm{~K}$. The instrumental resolution $\Delta E=1.30 \mathrm{meV}$ (FWHM) for all distributions except the one shown by crosses $(x)$ in (a). For crosses $\Delta E=12.0 \mathrm{meV}$. Filled circles ( $\bullet$ ) correspond to the $\mathrm{NaCl}$ phase. Open circles $(0)$ and crosses $(x)$ correspond to the $\mathrm{CsCl}$ phase of $\mathrm{NH}_{4} \mathrm{Br}$. Full curves are calculated using the octahedral jump model for $\tau=5 \mathrm{psec}$ and $\left\langle\boldsymbol{u}^{2}\right\rangle=0.125 \mathrm{~A}^{2}$. (Goyal and Dasannacharya 1979).

\subsection{Stochastic motions in metal-hydrogen systems and ferroelectrics through} incoherent quasielastic scattering

As indicated in $\$ 2.6$, neutrons are quasi-elastically scattered whenever hydrogen performs random motions. If the motion is purely translational as, for example, with hydrogen in metals, the scattered spectrum consists of a broadened line whose total intensity as a function of angle of scattering depends on the detailed geometry of the translational jumps of the proton (equation (5)). Such measurements were first reported for PdH by Skold and Nelin (1967) whose results, that hydrogen diffusion in $\mathrm{PdH}$ occurs by the jumps of hydrogen through octahedral interstitial sites in $\mathrm{Pd}$, have been further confirmed on single crystals of $\mathrm{PdH}_{0,03}$ by Rowe et al (1972). Figure 4 shows results of Rowe et al (1972) which also gives the average time between jumps to be 2.8 picosec. Similar experiments have also been performed on $\mathrm{V}, \mathrm{Nb}$ and $\mathrm{Ta}$ which show a more complicated jump pattern through tetrahedral interstitial sites (Lottner et al 1979; Springer 1977). More recently measurements have been greatly refined and tunneling states in $\mathrm{Nb} \mathrm{O}_{0.013} \mathrm{H}_{0.016}$ (Wipf et al 1981), hydrogen trapping cum diffusion in hydrogen storage material $\mathrm{Ti}_{1.2} \mathrm{Mn}_{1.8} \mathrm{H}_{3}$ (Hempelmann et al 1982) and multiple interstitial occupancy and jumps in $\mathrm{La} \mathrm{Ni}_{5} \mathrm{H}_{6}$ have been observed (Richter $e t$ al 1982). 

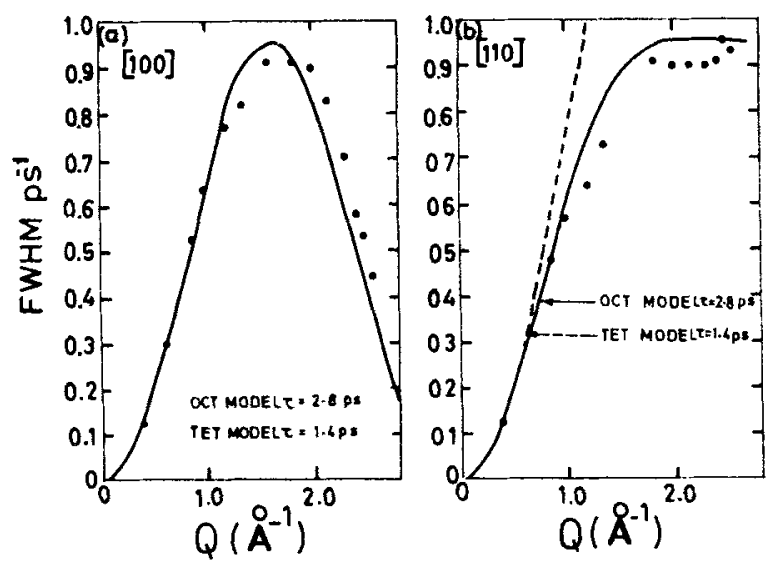

Figure 4. Variation of line-width of quasielastic line as a function of wavevector transfer for single crystal of $\mathrm{Pd} \mathrm{H}_{0.03}$ along (100) and (110) directions. (Rowe et al 1972)

Another class of experiments involves investigation of reorientation in solids with molecular groups containing hydrogen. In such a case the scattered spectrum consists of a sum of a purely elastic part (equation (6)) and a quasi-elastic part. An example of this class is ammonium sulphate. With two $\mathrm{NH}_{4}^{+}$ions in the molecule, it shows a phase transition at $-50^{\circ} \mathrm{C}$ from a paraelectric phase to a possibly ferrielectric phase. NMR results of $\mathrm{O}^{\prime}$ Reilly and Tsang (1967) suggested that in the paraelectric phase the two types of $\mathrm{NH}_{4}^{+}$ions have similar reorientation times $\tau_{\mathrm{I}}$ and $\tau_{\mathrm{II}}$. However, from similar NMR experiments Kydon et al (1969) concluded that $\tau_{\mathrm{I}}$ is smaller by almost an order of magnitude compared to $\tau_{\mathrm{II}}$ and that NMR is sensitive only to type II ions in the paraelectric phase. The situation was clarified with neutron quasielastic scattering experiments which demonstrated without doubt that in the paraelectric phase $\tau_{\mathrm{I}}$ and $\tau_{\mathrm{II}}$ are comparable. Using mixed salts like $\left[\left(\mathrm{NH}_{4}\right)_{0.16} \mathrm{~K}_{0.84}\right]_{2} \mathrm{SO}_{4}$ and pure $\left(\mathrm{NH}_{4}\right)_{2} \mathrm{SO}_{4}$ it was shown that at room temperature $\tau_{\mathrm{I}}=10 \cdot 4 \pm 3$ picosec and $\tau_{\mathrm{II}}=15 \cdot 8 \pm 1 \cdot 1$ picosec (Goyal and Dasannacharya 1978). The angular dependence of the elastic scattering (equation (6)) in the above two salts further showed that the experiments are consistent with reorientation about all four $\mathrm{N}-\mathrm{H}$ bonds in $\mathrm{NH}_{4}^{+}(\mathrm{I})$ being equally probable but that the reorientation probability about the four $\mathrm{N}-\mathrm{H}$ bonds in $\mathrm{NH}_{4}^{+}$(II) is not the same. In the ferroelectric phase, neutron experiments (Dasannacharya et al 1982) are consistent with NMR results of O'Reilly and Tsang (1967). Similar studies have been conducted on several other systems showing interesting phase transitions resulting from/in slowing down of reorientational motions (Springer 1977). We will however, now pass on to examples of inelastic scattering studies.

3.4 Ferroelectrics, superconductors, metal-hydrogen systems, hydrogenated metallic glasses through incoherent inelastic scattering

We have noted earlier that incoherent inelastic scattering arising out of one-phonon scattering relates to the polarisation weighted phonon frequency distribution function of hydrogen atoms under the reasonable assumption that scattering from other atoms can often be neglected (see table 1). Unlike infrared absorption and Raman scattering 
studies which are normally sensitive to phonons of long wavelength $\left(q \sim 10^{5} \AA^{-1}\right)$ only, the neutron scattering experiments provide information about phonons over the whole Brillouin zone $\left(q \sim 10^{8} \AA^{-1}\right)$. Experiments with single crystals can be utilised further to throw light on the nature of the polarisation vector associated with certain frequency modes (Thaper et al 1970). Though in suitable cases, these experiments have been used for obtaining the phonon frequency distribution function (Goyal et al 1972) more often these experiments have been performed to gain knowledge about the general features of the frequency distribution function leading to the understanding of phenomena like order-disorder transition, ferroelectricity, superconductivity etc. in hydrogenous systems. Some examples are given below:

We discussed the reorientations of $\mathrm{NH}_{4}^{+}$in ammonium sulphate earlier. $\left(\mathrm{NH}_{4}\right)_{2} \mathrm{SO}_{4}$ is a ferroelectric with $T_{c}$ of $-50^{\circ} \mathrm{C}$, but the isomorphic $\mathrm{K}_{2} \mathrm{SO}_{4}$ does not undergo a transition down to $4.2^{\circ} \mathrm{K}$. It is therefore of interest to systematically examine the role of replacing $\mathrm{NH}_{4}^{+}$with $\mathrm{K}^{+}$. Results of inelastic scattering experiments at $110^{\circ} \mathrm{K}$, in the ferroelectric phase of $\left(\mathrm{NH}_{4}\right)_{2} \mathrm{SO}_{4}$ are shown in figure 5 (Chandra et al 1976). The two prominent peaks at 390 and $350 \mathrm{~cm}^{-1}$ are associated with the librational modes of the two ammonium ions $\mathrm{NH}_{4}^{+}$(I) and $\mathrm{NH}_{4}^{+}$(II) in the unit cell (Schlemper and Hamilton 1966). On the other hand. the spectrum for $\left[\left(\mathrm{NH}_{4}\right)_{0.16} \mathrm{~K}_{0.84}\right]_{2} \mathrm{SO}_{4}$ shows only one group of librational frequencies at $350 \mathrm{~cm}^{-1}$. As the concentration of $\mathrm{NH}_{4}^{+}$increases beyond $50 \%$ the frequency at $390 \mathrm{~cm}^{-1}$ starts building up and finally grows into full intensity for the pure ammonium salt. These measurements are interpreted to mean that $\mathrm{NH}_{4}^{+}$ion first preferentially goes to one type of site, $\mathrm{NH}_{4}^{+}$(II), till most of that site is occupied. In combination with dielectric measurements (Sawada et al 1975) which show that $\left[\left(\mathrm{NH}_{4}\right)_{\gamma} \mathrm{K}_{1-x}\right]_{2} \mathrm{SO}_{4}$ shows ferroelectric properties only for $x \geq 0-45$, the

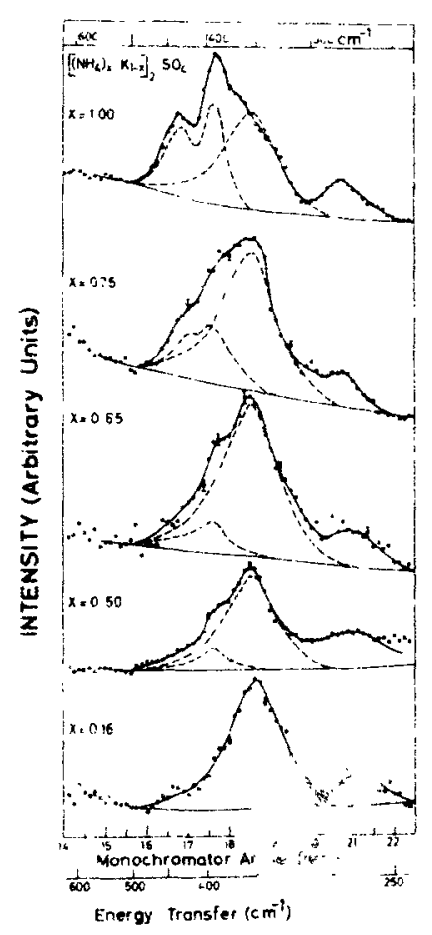

Figure 5. Spectra of incoherent inelastically scattered neutrons for $\left[\left(\mathrm{NH}_{4}\right)_{x} \mathrm{~K}_{1-x}\right]_{2} \mathrm{SO}_{4}$ for different $x$ values at $110 \mathrm{~K}$ (Chandra et al 1976). 
neutron results can be interpreted to mean that the presence of $\mathrm{NH}_{4}^{+}$(I) is necessary for the onset of ferroelectricity in this compound. These results also indicate that librational modes of $\mathrm{NH}_{4}^{+}$(II) are not directly involved in the ferroelectric transition, since the $\mathrm{NH}_{4}^{+}$(II) frequency in the ferroelectric sample with $x>0.45$ are identical to those in the paraelectric samples with $x<0.45$.

In addition to such measurements (by forming isomorphic mixed salts) studies have also been conducted by deuterating or partially deuterating the samples in order to identify the role and coupling of a particular hydrogen atom in a compound. Such studies have been carried out on a number of organic compounds, polymers, amino acids, etc (Reynolds and White 1969; Twisleton and White 1972; Thaper et al 1981). Some measurements have also been made to study the role of hydrogen adsorbed on nickel (Stockmeyer et al 1975), platinum black (Howard et al 1977) and in zeolites (Stockmeyer and Monkenbusch 1980).

Metal hydrogen systems like $\mathrm{Zr} \mathrm{H}_{2}, \mathbf{Y H}_{2}, \mathrm{NbH}_{x}, \mathrm{VH}_{x}, \mathbf{P d H}_{x}$, etc. have been extensively studied by inelastic scattering methods (for a review see Dasannacharya 1980). Systematics of the vibrational frequency against metal hydrogen distance for hydrogen in octahedral and tetrahedral sites have been evolved (Hunt and Ross 1976). Thus, it is now possible to predict the nature of site occupancy by knowing the vibrational frequency. More recently, measurements have also been carried out on metallic glasses (Rush et al 1980, Kajitani et al 1982) loaded with hydrogen. The results of Ti Cu H (Rush et al 1980) in the crystalline and glassy state are shown in figure 6. The large broadening of the vibrational density of states in the glassy state is believed to arise because of a large variation in the local environment of hydrogen atoms as compared to that in the crystalline materials where hydrogen occupies the tetrahedral interstitial site.

Finally, this technique has also been used to examine the role of hydrogen, and possibly that of optical phonons in superconductivity of $\mathrm{Th}_{4} \mathrm{H}_{15}$ and $\mathrm{PdH}_{x}$ (Dietrich $e t$ al 1977; Rowe et al 1974).

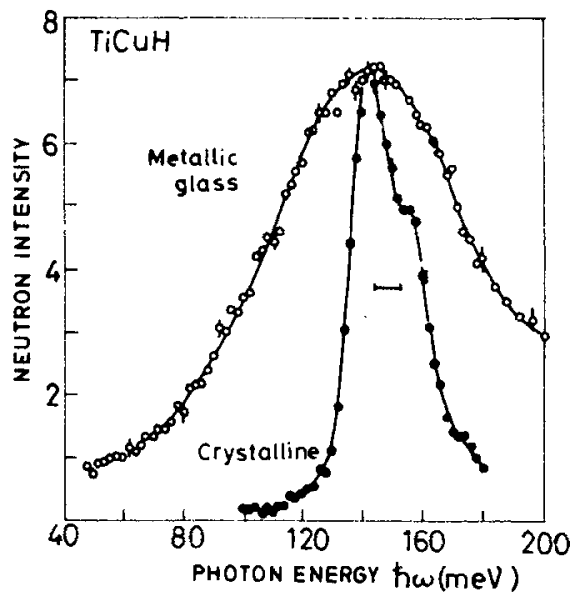

Figure 6. Scattered neutron spectra measured at $78 \mathrm{~K}$ for crystalline $\mathrm{TiCuH}_{0.93}(\bullet)$ and amorphous $\mathrm{Ti} \mathrm{Cu} \mathrm{H}_{1,3}(0)$. The energy resolution (FWHM) near the peak is indicated by horizontal bar (Rush et al 1980). 


\subsection{Molecular crystals, polymers through coherent inelastic scattering}

Coherent scattering measurements are the only known method for detailed measurement of phonon dispersion curves. In addition to the normal difficulty of getting a single crystal, in the case of hydrogenous substances one may have the additional problem of obtaining deuterated samples. However, having overcome these difficulties one stands the chance of obtaining information which is unique and extremely detailed in nature. Successful experiments have been carried out on inorganic crystals (Smith et al 1969; Vagelatos et al 1975), molecular crystals (Chaplot 1983), metal-hydrogen systems (Springer 1978) and polymers (King 1974). As an example, we show the dispersion curve for polyethylene measured by Feldkamp et al (1968) (figure 7). We, however, also mention in passing that these measurements are difficult and time-consuming and have been successfully attempted only recently.

\subsection{Polymers and bio-molecules through small angle neutron scattering}

Small angle neutron scattering (SANS) technique, which examines the sample with a typical spatial resolution of $\sim 100 \AA$, is an excellent tool for determining the configuration of polymer chains and that of large size molecules of biological interest by measuring their radius of gyration. This is in fact the only technique for examining the chain configurations in solid polymers, polymer melts and concentrated polymer solutions. The other available techniques (small angle $\mathrm{x}$-ray scattering or light scattering ) can be used only for dilute polymer solutions (Higgins and Stein 1978).

The importance of SANs technique in the study of hydrogen and hydrogenous materials emerges from the fact that hydrogen and deuterium have quite different neutron coherent scattering amplitudes (see table 1). In a given hydrogenous material, it is thus possible to enhance the contrast between one set of molecules as compared to the other by deuterating them. For example, in a study of biological molecules where sample is often dissolved in water, the contrast between the sample molecules and the solvent molecules can be varied in a continuous way by using mixtures of $\mathrm{H}_{2} \mathrm{O}$ and $\mathrm{D}_{2} \mathrm{O}$. The major components of biological materials are proteins, nucleic acids, carbohydrates and lipids. The scattering densities of these components are all different, but are between the scattering densities of $\mathrm{H}_{2} \mathrm{O}$ and $\mathrm{D}_{2} \mathrm{O}$. Thus a variation in contrast

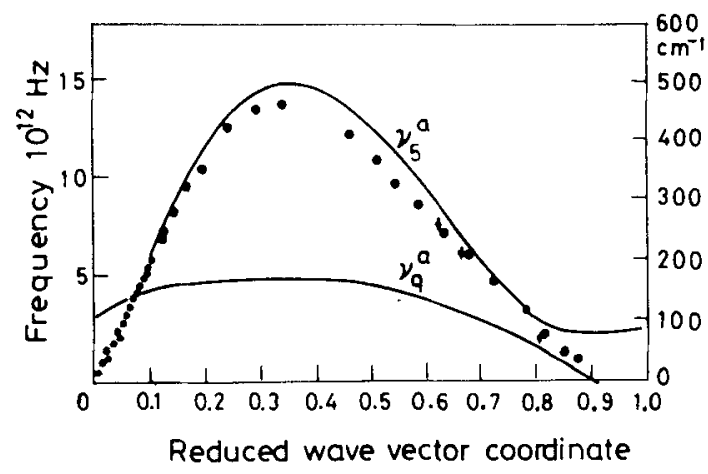

Figure 7. Comparison of experimental and calculated dispersion curves for deuterated polyethylene (Feldkamp et al 1968). 
greatly helps in determining the spatial arrangements of various components in the biological material. This method has been successfully used to separate out RNA and protein part of the structure in spherical viruses and ribosomes (Zaccai 1978). Figure 8 shows the organization of protein and RNA in turnip yellow mosaic virus (TYMv) and cucumber mosaic virus (CMV) as obtained using sANs technique (Jacrot et al 1977). The shaded regions in this figure correspond to RNA, and show that penetration of RNA in the protein shell is larger in CMV than that in the case of TYMV.

For solid polymers samples, where all chains are identical, contrast between a given chain and the neighbouring chains can be enhanced by deuterating that chain. Scattered neutron intensity in SANS experiment on such a polymer sample is decided by the concentration of the labelled chains and by the contrast factor $\left|b_{s}-b_{h}\right|^{2}$, where $b_{s}$ and $b_{h}$ are the scattering amplitudes of the monomers in the labelled chains and in the matrix chains respectively (equation (7)). Though earlier studies (Cotton et al 1974) on polymer systems were carried out using low $(<1 \%)$ concentration of labelled chains, it is possible to gain in intensity by using higher (upto $50 \%$ ) concentration of labelled chains without spoiling the quality of information obtained (Akcasu et al 1980; Goyal et al 1983). For example, in their sans studies on solid polystyrene, Tangari et al (1980) used $30 \%$ tagging and obtained results similar to those obtained by Cotton et al (1974) using low concentration tagging. This is shown in figure 9 , where the measured radius of gyration $R_{w}$ for polystyrene has been plotted as a function of its molecular weight $M_{w}$

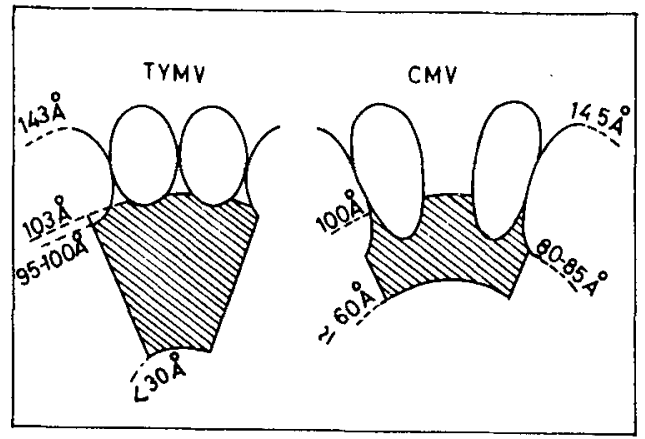

Figure 8. Sketch of the organisation of protein and RNA in turnip yellow mosaic virus and cucumber mosaic virus as obtained using neutron scattering data. The shaded area corresponds to RNA (Jacrot et al 1977).

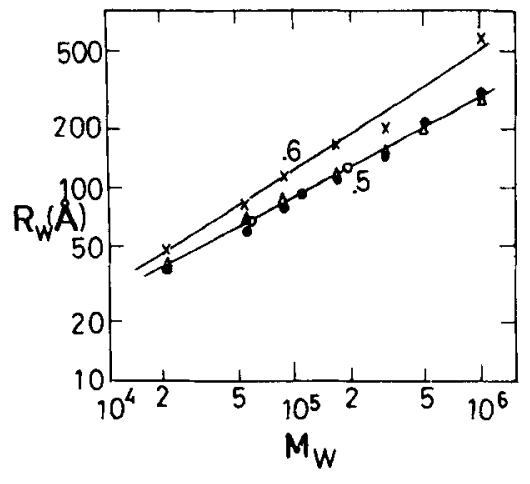

Figure 9. Radius of gyration $R_{w}$ as a function of molecular weight $M_{w}$ for polystyrene. Solid ( $(\bullet)$ and open (o) circles correspond to solid polystyrene. Filled circles were obtained using low concentration tagging and open circles were obtained using high concentration tagging. Crosses $(x)$ correspond to polystyrene in $\mathrm{CS}_{2}$ and $(\Delta)$ correspond to polystyrene in cyclohexane at $36^{\circ} \mathrm{C}$ (Tangari et al 1980). 
for different experimental conditions. Filled circles (๑) and open circles (o) in this figure correspond to solid polystyrene and have been obtained using low (Cotton et al 1974) and high (Tangari et al 1980) concentration tagging. It is found that for solid polystyrene $R_{w}$ varies as $M_{w}^{0.5}$, beautifully confirming, Flory's (1953) prediction based on his random coil model for solid polymers. Figure 9 also shows results of SANS experiments for polystyrene dissolved in a good solvent $\left(\mathrm{CS}_{2}\right)$ and a theta solvent (cyclohexane at $36^{\circ} \mathrm{C}$ ) (Cotton et al 1974). For good solvents $(\times), R_{w}$ varies as $M_{w}^{0.6}$ whereas for theta solutions $(+), R_{w} \sim M_{w}^{0.5}$. During last decade, a number of polymer systems have been studied using SANS technique (Higgins 1979). Dynamics of these chains have also been investigated through quasielastic scattering (Higgins 1979).

\section{Concluding remarks}

Neutrons are the single most powerful probe for investigating hydrogenous substances. It is the purpose of this review to illustrate this through examples of different neutron scattering techniques and a wide variety of materials. Though it has not been possible to include all the methods, it is hoped that the main point has been conveyed.

\section{References}

Akcasu A Z, Summerfield G C, Jahshan S N, Han C C, Kim C Y and Yu H J 1980 Polymer Sci. 181963 Bansal M L, Sahni V C and Roy A P 1979 J. Phys. Chem. Solids 40109

Bhakay-Tamhane S N, Sequeira A and Chidambaram R 1980 Acta Crystallogr. B36 2925

Chandra P P, Dasannacharya B A, Goyal P S, Iyengar P K, Rao K R, Thaper C L and Venkatesh A H 1976 Phys. Lett. A57 463

Chaplot S L 1983 Nuclear Physics and Solid State Phys. India A26 (in press)

Cotton J P, Decker D, Benoit H, Farnoux B, Higgins J, Jannink G, Ober R, Picot C and des Coiseaux J 1974 J. Macromol. 7863

Dachs H 1978 in Topics in current physics Vol. 6 (Berlin: Springer-Verlag)

Dasannacharya B A 1980 Hydrogen in metals (Bombay: Dept. Atomic Energy) pp 59

Dasannacharya B A, Richter D and Subramanian R 1982 Nuclear Physic's and Solid State Physics (Iniala) C25 (in press)

de Graff L A, Chr. Steenbergen and Heidemann A 1980 Physica B101 209

Dietrich M, Reichardt W and Rietschel H 1977 Solid State Commun. 21603

Feldkamp L A, Venkataraman G and King J S 1968 Inelastic scattering of neutrons (Vienna: IAEA) pp 159

Flory P J 1953 Principles of chemistry, (Ithaca, New York: Cornell University Press)

Goyal P S, Dasannacharya B A, Thaper C L and Iyengar P K 1972 Phys. Status. Solidi. B50 701

Goyal P S and Dasannacharya B A 1978 J. Cnem. Phys. 682430

Goyal P S and Dasannacharya B A 1979 J. Phys. C12 219

Goyal P S, King J S and Summerfield G C 1983 Polymer 24131

Hempelmann R, Richter D and Heidemann A 1982 J. Less Common Metals 88343

Higgins J 1979 Treatise on materials science and iechnology Vol. 15, (ed.) G Kostorz (New York :Academic Press) pp 381

Higgins J S and Stein R S 1978 J. Appl. Crystallogr. 11346

Howard J, Waddington T C and Wright C J 1978 J. Chem. Soc. Faraday II 731768

Hunt D G and Ross D K 1976 J. Less Common Metals 4969

Jacrot B, Chauvin C and Witz J 1977 Nature (London) 266417

Kajitani T, Kaneko H and Hirabayashi M 1981 Science Report of the Research Institutes, Tohuku University, Sendai, Japan A29 210

King J S 1974 Spectroscopy in biology and chemistry (eds) S H Chen and S Yip (New York: Academic Press) pp 235

King J S 1980 Methods of experimental physics (New York: Academic Press) Vol. A16, pp 480 
Kostorz G and Lovesey S W 1979 Treatise on materials science and technology, (ed.) G Kostorz (New York: Academic Press) Vol. 15

Kydon D W, Petch H E and Pintar M 1969 J. Chem. Phys. 51487

Leadbetter A J, Richardson R M, Dasannacharya B A and Howells W S 1976 Chem. Phys. Lett. 39501

Lottner V, Buchenau V and Fitzgerald W J 1979 Z. Phys. B35 35

O'Reilly D E and Tsang T 1967 J. Chem. Phys. 461291

Oxton I A and Knopp O 1977 Chem. Phys. Lett. 49560

Reynolds P A and White J W 1969 Disc. Faraday Soc. 48131

Richter D, Hempelmann R and Vinhas L A 1982 J. Less Common Metals 88353

Rowe J M, Rush J J, de Graff L A and Fergusen G A 1972 Phys. Rev. Lett. 291250

Rowe J M, Rush J J, Smith H G, Mostoller M and Flotow H E 1974 Phys. Rev. Lett. 331297

Rush J J, Rowe J M and Maeland A J 1980 J. Phys F10 L283

Sawada A, Ohya S, Ishibashi Y and Takagi Y 1975 J. Phys. Soc. (Jpn) 381408

Schlemper E O and Hamilton W C 1966 J. Chem. Phys. 444498

Skold K and Nelin G 1967 J. Phys. Chem. Solids 282369

Smith H G, Traylor J G and Reichardt W 1969 Phys. Rev. 1811218

Springer T 1972 Springer-tracts in modern physics (Berlin: Springer-Verlag) Vol. 64

Springer T 1977 in Topics in current physics (eds) S W Lovesey and T Springer (Berlin: Springer-Verlag) Vol. 3, pp 255

Springer T 1978 in Topics in applied physics (eds) G Alefeld and J Volkl (Berlin: Springer-Verlag) Vol. 28, pp 75

Stockmeyer R, Conrad H M, Renouprez A J and Fouilloux P 1975 Surface Sci. 49549

Stockmeyer R and Monkenbusch 1980 Paper presented at the 5th International Conference on Zeolites, Napoli.

Tangari C, Summerfield G C, King J S, Berliner R and Mildner D F R 1980 Macromolecules 131546

Thaper C L, Dasannacharya B A, Sequeira A and Iyengar P K 1970 Solid State Commun. 8497

Thaper C L, Sinha S K and Dasannacharya B A 1982 Neutron scattering 1981 (ed.) John Faber Jr (New York: American Institute of Physics) pp 249

Twisleton J F and White J W 1972 Neutron inelastic scattering (Vienna: IAEA) pp 301

Twisleton J F and White J W 1972 Polymer 1340

Vagelatos N, Rowe J M and Rush J J 1975 Phys Rev. B10 4522

Wipf H, Magerl A, Shapiro S M, Satija S K and Thomlinson W 1981 Phys. Rev. Lett. 14949

Zaccai G 1978 in Topics in current physics (ed.) H Dachs (Berlin: Springer-Verlag) Vol. 6, pp 243 